\title{
Conjeturación del teorema del valor medio para derivadas: Un acercamiento desde la detección de invariantes en dispositivos móviles con GeoGebra
}

\section{Conjecturing process for the mean value theorem for derivatives: An approach from the detection of invariants in mobile devices with GeoGebra}

\author{
DOI: http://dx.doi.org/10.17981/cultedusoc.12.1.2021.05
}

Recibido: 20 de abril de 2020 Aceptado: 04 de septiembre de 2020 Publicado: 1 de diciembre de 2020

Vladimir Ballesteros-Ballesteros (D)

Fundación Universitaria Los Libertadores. Bogotá, D.C. (Colombia)

vladimir.ballesteros@libertadores.edu.co

Óscar Iván Rodríguez-Cardoso (1)

Fundación Universitaria Los Libertadores. Bogotá, D.C. (Colombia)

oscar.rodriguez@libertadores.edu.co

Sébastien Lozano-Forero (D)

Fundación Universitaria Los Libertadores. Bogotá, D.C. (Colombia)

sebastien.lozano@libertadores.edu.co

Para citar este artículo:

Ballesteros-Ballesteros, V., Rodríguez-Cardoso, Ó. y Lozano-Forero, S. (2021). Conjeturación del teorema del valor medio para derivadas: Un acercamiento desde la detección de invariantes en dispositivos móviles con GeoGebra. Cultura, Educación y Sociedad, 12(1), 19-84. DOI: http://dx.doi.org/10.17981/cultedusoc.12.1.2021.05

\section{Abstract}

Este artículo presenta los resultados de un proyecto de investigación cuyo objetivo fue describir el papel mediador de la aplicación móvil "Calculadora Gráfica" de GeoGebra sobre los procesos de conjeturación del teorema del valor medio para derivadas mediante la detección de invariantes a través de herramientas de arrastre, combinando geometría dinámica con cálculo infinitesimal. A través de un estudio de caso cualitativo, que involucró estudiantes de Ingeniería Aeronáutica, se dinamizaron los esfuerzos investigativos con el propósito de validar la hipótesis relacionada con una influencia positiva de una estrategia de aprendizaje móvil sobre el proceso de conjeturación en un curso de Cálculo Diferencial. Los resultados obtenidos permitieron evidenciar avances significativos en la conjeturación del teorema mencionado para la resolución de problemas en ingeniería y se discute cómo este tipo de recursos digitales, a través de un entorno de geometría dinámica en dispositivos móviles, puede servir como mediación para favorecer el aprendizaje del cálculo.

Palabras clave: Aprendizaje móvil; conjeturación; GeoGebra; teorema del valor medio para derivadas

\section{Resumen}

This article presents the results of a research project whose main objective was to describe the mediating role of GeoGebra's "Graphing Calculator" mobile application on the conjecturing processes of the mean value theorem for derivatives by the use of some dragging tools, which combines dynamic geometry and infinitesimal calculus. By means of a qualitative case study, involving students from aeronautical engineering, research efforts were carried out looking forward to get evidence that allow the judgement of a hypothesis involving a positive influence of a mobile learning strategy on conjecturing processes in the context of a calculus course. The results obtained allowed us to conclude significant advances in the conjecturing process of the abovementioned theorem for the solving-problems process in engineering. The discussion of how this type of digital resources, through a dynamic geometry environment in mobile devices, could favor the learning of calculus, is also addressed.

Keywords: Mobile learning; conjecturing process; GeoGebra; mean value theorem for derivatives 


\section{INTRODUCCIÓN}

Existe hoy un nuevo escenario de trabajo con medios digitales e informáticos que abarca desde la representación, o modelamiento, de escenarios espaciales, hasta la posibilidad de diseñar y generar lugares a partir de estructuras de datos nuevas y complejas. Esto ha sido posible gracias al desarrollo de técnicas y metodologías de procesamiento extremadamente potentes para la visualización gráfica, llegando a permear el campo de la educación a través de la creación de nuevas herramientas de difusión, de estructuras para la creación de conocimiento y aprendizaje; en particular, se han implementado nuevas ideas y líneas de acción relacionadas con los diversos tipos de tecnologías establecidas, proporcionando un lugar preponderante para el aprendizaje móvil o m-learning (Crompton, Burke, Gregory \& Grabe, 2016; Joo-Nagata, Martínez, García-Bermejo \& García-Peñalvo, 2017).

El uso de dispositivos móviles como herramientas tecnológicas es fundamental en lugares de trabajo, dentro o fuera de las aulas en el contexto de la educación superior, proporcionando instrucción virtual, aprendizaje por medio de teléfonos móviles y cualificación a través de Internet. Estas herramientas, correctamente implementadas, pueden cambiar la dinámica tradicional de clase y proponer una definición alternativa de educación que reconozca en el estudiante, más autonomía y más independencia, al permitir que éste aprenda en casa, en el lugar de trabajo o durante un viaje, en cualquier momento y en cualquier lugar, dado que minimiza limitaciones e ineficiencias (Hamidi \& Chavoshi, 2018).

La capacidad de aprender en cualquier momento y en cualquier lugar, es una de las características del e-learning (base para el desarrollo del m-learning), entendido como un tipo de aprendizaje electrónico que se implementa a través de tecnologías móviles como Asistentes Digitales Personales (PDA), teléfonos celulares, reproductores de audio, libros electrónicos, entre otros. El m-learning puede verse como una evolución natural del e-learning, permitiendo a los estudiantes y usuarios desarrollar un proceso de aprendizaje a través de la tecnología móvil (Holmes \& Gardner, 2006; Sánchez, Olmos \& GarcíaPeñalvo, 2013; 2016; Pegrum, 2014; Karimi, 2016; Hamidi \& Chavoshi, 2018). Las pruebas empíricas indican que el aprendizaje móvil puede ser un instrumento útil que permite a los estudiantes aprender en muchas áreas de conocimiento, entre ellas la educación, los negocios y las tecnologías de la información (Ozdamli, 2012; Al-Emran, Mezhuyev \& Kamaludin, 2018).

Por otra parte, GeoGebra ha generado una profunda transformación en la enseñanza de las matemáticas. En este Ambiente de Geometría Dinámica (AGD), los estudiantes puede mover o arrastrar un punto libre elegido y explorar cómo cambian puntos construidos y otros objetos; esta característica es simple pero muy atractiva y efectiva en la educación matemática debido a que, en lugar de explorar figuras geométricas estáticas, los estudiantes pueden explorar figuras estrechamente relacionadas obtenidas arrastrando algunos puntos móviles y, a partir de allí, concluir algunas propiedades sobre ellos (Selaković, Marinković \& Janičić, 2019). También, proporciona un contexto donde las propiedades geométricas, que aparecen como invariantes bajo propiedades de arrastre, pueden percibirse de manera visual y kinestésica, y vincularse de nuevo a los fundamentos teóricos subyacentes incrustados en el sistema (Baccaglini-Frank, 2019). El principio de arrastre 
se encuentra en el centro del diseño de un AGD típico y abarca no solo la variación sino también los invariantes y relaciones entre ellos, vinculando explícitamente la capacidad de manipulación de las figuras con propiedades geométricas definidas, en particular, esos invariantes y su identificación a través de arrastre (Leung, Baccaglini-Frank \& Mariotti 2013; Mariotti, 2015).

Un antecedente notable sobre la vinculación de AGD a los procesos de demostración se relaciona con los resultados de su experimento de enseñanza, desarrollado en el curso de Geometría Plana de una Licenciatura en Matemáticas, desarrollado durante varios semestres, abordando temas como las relaciones entre puntos, rectas, planos, ángulos, propiedades de los triángulos, cuadriláteros, círculos y relaciones de congruencia y similitud mediado por el software "Cabri Geometry" (versión 2.1.1) con la intención de favorecer el aprendizaje de los estudiantes. Entre sus hallazgos, se evidencia que el AGD resulta ser una herramienta que facilita la comprensión de todas las condiciones expuestas en diferentes problemas, descubrir si han impuesto propiedades adicionales o restrictivas, comprender las relaciones de dependencia involucradas y, por lo tanto, la lógica detrás de una declaración dada en forma de implicación (Camargo, Samper \& Perry, 2007). Otros resultados de investigación más recientes admiten las funcionalidades y beneficios de vincular dispositivos táctiles que se hacen cada vez más populares en el aprendizaje de la geometría, tales como teléfonos móviles y tabletas en el contexto de la geometría dinámica (Cheema, Gulwani \& LaViola, 2012; Ehmann, Gerhauser, Miller \& Wassermann, 2013).

Para el desarrollo de la investigación, se implementó la aplicación para dispositivos móviles "Calculadora Gráfica" de GeoGebra (versión 6.0.619.0). Esta aplicación móvil se enmarca dentro de las tecnologías digitales y cuenta con el respaldo de comunidades académicas interesadas en apoyar procesos de aprendizaje y enseñanza de las matemáticas y disciplinas relacionadas; GeoGebra es una plataforma de libre acceso y está compuesta por herramientas fáciles de manejar, admite geometría dinámica, permite múltiples representaciones de conceptos, permite guardar y exportar archivos de salida en múltiples formatos como ggb, html, xml, entre otros; facilita la inserción de imágenes, puede ser enlazado con paquetes tipográficos especiales como LaTeX, tiene capacidades de prueba automatizadas, conlleva a usar varios lenguajes en sus menús y sus comandos a través de la producción de materiales didácticos, como hojas de trabajo dinámicas independientes, destacando applets interactivos de Java que podrían publicarse en un sitio web y existe toda una comunidad global activa de usuarios que proporcionan material didáctico y soporte técnico (Escuder \& Furner, 2011; Velichova, 2011; Majerek, 2014; Wassie \& Zergaw, 2019).

\section{REVISIÓN DE LA LITERATURA}

La necesidad de promover el razonamiento matemático y la demostración en todos los niveles escolares es ampliamente reconocida en el contexto global, destacando que estos aspectos deben ser tenidos en cuenta para el diseño curricular en matemáticas (Boero, Fenaroli \& Guala, 2018). Una de las razones para considerar el proceso de demostración como un tema importante en educación matemática consiste en reconocer que la demostración está estrechamente relacionada con la actividad de argumentación involucrada en la resolución de un problema (Boero et al., 2018; Pedemonte 2007; 2018). Los libros de texto 
de matemáticas muestran los procesos de demostración como el resultado de definiciones, axiomas y teoremas vistos durante el desarrollo teórico, sin embargo, no reflejan las ideas que llevaron a los matemáticos a producir tales pruebas (Karunakaran, 2018). Así, la relación cognitiva entre la intuición y la demostración es compleja y para los estudiantes puede resultar complejo encontrar justificaciones para explicar lo que parece evidente cuando resuelven un problema, por ejemplo, de índole geométrico (Mariotti y Pedemonte, 2019).

Hanna (1995) se refiere a dos usos que puede tener la demostración en educación matemática: uno, es la prueba con el único sentido de demostrar la validez de una proposición y, el otro, es la prueba por lograr no solamente la demostración de su veracidad sino también la explicación del fenómeno matemático subyacente. Para ilustrar esta situación, se revisa la suma de los $n$ primeros números naturales, dada por la expresión (1) que se puede demostrar o probar fácilmente por inducción matemática. Sin embargo, esta prueba no explica el fenómeno en sí mismo, como lo indica Hanna (1995) "No da al estudiante idea alguna de por qué es verdad" (p. 48). Sin embargo, en una prueba explicativa se puede llegar a dotar de sentido la proposición sumando dos veces los primeros números naturales de forma simétrica o por medio de representaciones geométricas usando cuadrados de una unidad de lado que completan un rectángulo cada vez más grande que luego es dividido a la mitad (Tabla 1).

$$
S_{n}=1+2+3+\cdots+n=\frac{n(n+1)}{2}
$$

TABLA 1.

Ejemplo de argumentos explicativos de veracidad de la conjetura $S_{n}=\frac{n(n+1)}{2} \forall n \in N$.

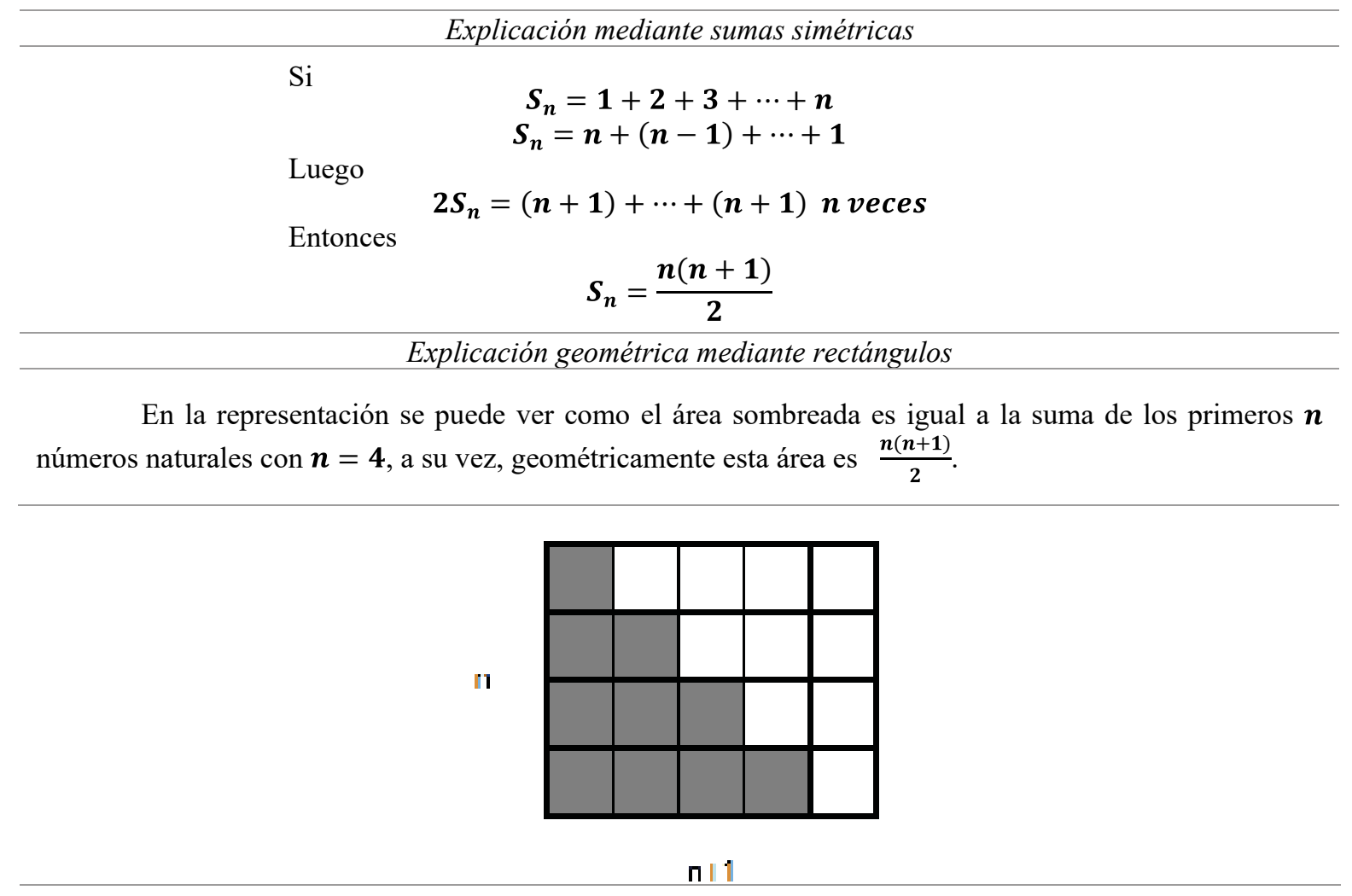

Fuente: Elaboración propia 
De acuerdo con Hanna (1995), incluso un matemático experimentado prefiere las pruebas o demostraciones de tipo explicativo y, desde luego, para los maestros de matemáticas es aún más importante tomarse el tiempo para buscar pruebas o demostraciones que promuevan la comprensión, es decir, que puedan dar lugar a la explicación, no solo un conocimiento del qué, sino también conocimiento del por qué. Hanna (2018) presenta avances importantes en las nociones de explicación matemática y de prueba explicativa, afirmando que no pueden ser capturadas por un solo modelo. Adicionalmente, señala que los posibles modelos emergentes para la explicación, en matemáticas, sin duda tomarían en consideración enfoques como el uso de analogías, ejemplos y lógica basada en reglas, así como también evaluarían los roles de la intuición y la visualización, al tiempo que recrearían criterios variables para la validez de una prueba. Recomienda, además, que los educadores matemáticos deberán seguir recurriendo a múltiples recursos, confiar en su juicio y ser pragmáticos al tratar de identificar o construir pruebas que sean suficientemente explicativas para cumplir sus objetivos pedagógicos; en este sentido, cuando una conjetura se construye a través de una argumentación constructiva, la demostración puede ser más accesible para los estudiantes (Mariotti y Pedemonte, 2019).

Reafirmando esta idea, Mariotti y Pedemonte (2019) muestran que los estudiantes pueden llegar a argumentar una conjetura a través de un proceso de construcción con base en resultados anteriores, para luego hilar coherentemente una prueba. Sin embargo, algunas veces se puede llegar a la conjetura por medio de evidencia empírica o intuición sin tener un proceso de construcción previo, en este caso consolidar una demostración involucrará una mayor complejidad de los estudiantes, quienes deberán elaborar una estructura argumentativa para justificar su conjetura, luego podrán obtener la prueba en referencia a esta argumentación, sin embargo, llegar a una demostración por esta vía es mucho más difícil en comparación con el primer proceso.

\section{Metodología}

La investigación se implementó a través de un estudio de caso cualitativo desde la perspectiva de Creswell (2017) aplicado a cinco estudiantes del programa de Ingeniería Aeronáutica. Cada uno de los cinco estudiantes matriculó el espacio académico Cálculo Diferencial de acuerdo con el procedimiento institucional para tal fin, con la posibilidad de seleccionar libremente el grupo de adscripción, y sin la existencia de grupo puntual de observación. Así, el proceso de selección de los estudiantes se llevó a cabo a través de un muestreo aleatorio simple dentro de la población de estudiantes de primer y segundo semestre del programa que debían cursar este espacio académico. 
El tratamiento experimental desarrollado con el grupo estuvo compuesto por siete problemas y una construcción geométrica dinámica cuyo principal objetivo fue inducir el proceso de conjeturación del Teorema del Valor Medio para Derivadas (TVMD) a través de la identificación de invariantes observados por medio de arrastre y manipulación de objetos matemáticos en dispositivos móviles como teléfonos inteligentes, tabletas o iPads a través de la interacción con la ACGG. Para el desarrollo de las actividades, cada estudiante disponía de un dispositivo móvil con la aplicación y una guía de trabajo contentiva de los siete problemas que debía desarrollar. Los estudiantes se dispusieron espacialmente en el aula de clase en forma de mesa redonda para poder interactuar con sus pares mediante el diálogo y socialización de sus hallazgos; se les pidió interrumpir cualquier tipo de conexión a Internet para reducir posibles sesgos en el ejercicio. El profesor desempeñó un papel secundario durante las cinco sesiones de dos horas que duró el estudio de caso y se limitó únicamente a capturar registros audiovisuales de la experiencia, por lo tanto, no intervino activamente en el proceso de los estudiantes; todo lo encontrado por ellos fue desde su propia interacción con la ACGG y con sus pares.

A continuación, se muestran los problemas abordados en la intervención, así como la construcción geométrica dinámica en la ACGG.

\section{Problema 1:}

Responda la siguiente pregunta, desde sus conocimientos previos, usando la aplicación "Calculadora Gráfica” de GeoGebra en caso de considerarlo necesario.

1.1. ¿Cree usted que, si dos corredores en una misma carrera parten del mismo punto y al mismo tiempo, y llegan al mismo tiempo a la meta, en todo momento tuvieron la misma velocidad? Justifique su respuesta.

1.2. ¿Cree usted que, al menos en algún instante de tiempo durante esa competencia, tuvieron la misma velocidad? Justifique su respuesta.

1.3. Si lo considera necesario, represente la situación en la aplicación móvil de GeoGebra.

1.4. Comparta sus conclusiones con el grupo de trabajo.

El problema 1 se diseñó como diagnóstico, de tal manera que se pudiera recoger información acerca de los conceptos previos que dinamizarían la interacción de los estudiantes con el TVMD mediante un problema de la física. También se pretendía identificar si algún estudiante vinculaba la ACGG para el desarrollo del problema. De acuerdo con el orden de la actividad, enseguida los estudiantes debían realizar la construcción 1 (Tabla 2). 
TABLA 2.

Construcción Geométrica dinámica en la ACGG

Paso 1: Dibuje la función $\mathbf{f}(\mathbf{x})=\operatorname{sen}(\mathbf{x})$. Se sugiere color negro.

Paso 2: Defina tres deslizadores y etiquételos como $\mathbf{a}=-\mathbf{1}, \mathbf{b}=\mathbf{2}$ y $\mathbf{c}=\mathbf{1}$

Paso 3: Determine los puntos $\mathbf{A}=(\mathbf{a}, \mathbf{0}), \mathbf{B}=(\mathbf{b}, \mathbf{0})$ y $\mathbf{C}=(\mathbf{c}, \mathbf{0})$, sobre el eje $\mathbf{X}$. Por conveniencia use color azul para A y B, y rojo para C.

Paso 4: Con la herramienta "Perpendicular" trace rectas perpendiculares al eje $\mathbf{X}$ que pasen por los puntos A, B y C

Paso 5: Usando la herramienta "Intersección" defina los puntos de intersección de cada una de las rectas del paso 4 con la función $\mathbf{f}(\mathbf{x})$, etiquételos como se ve y configúrelos con los colores sugeridos.

Paso 6: Con la

herramienta "segmento", trace el segmento $\overline{\mathbf{D F}}$ en color verde teniendo en cuenta que $\mathbf{D}=(\mathbf{a}, \mathbf{f}(\mathbf{a}))$, $\mathbf{F}=(\mathbf{b}, \mathbf{f}(\mathbf{b}))$.

Paso 7: Oculte todos los elementos de la construcción excepto la función $\mathbf{f}(\mathbf{x}), \overline{\mathbf{D F}}$, y los puntos A, B, C, D, E, F.

Paso 8: Trace la recta tangente a $\mathbf{f}(\mathbf{x})$ que pasa por $\mathbf{E}$ (en adelante $\mathbf{L} \mathbf{1}$ la opción "Tangentes".

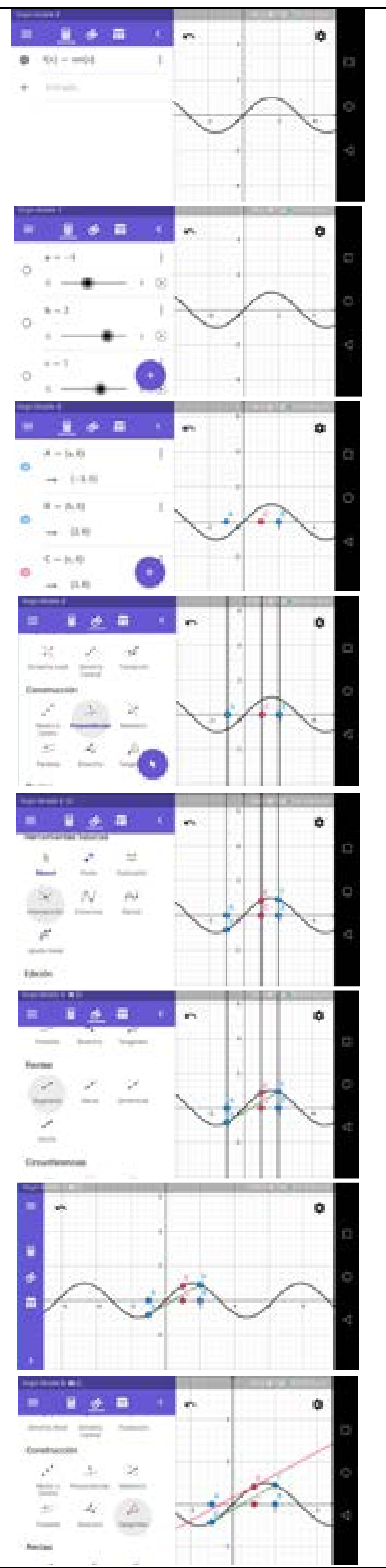

Nota: Paso a paso de la primera construcción. Fuente: Elaboración propia. 
Los problemas 2 al 7, fueron diseñados para que los estudiantes interactuaran con la construcción 1 mediante el uso de la ACGG desde sus dispositivos móviles, y se presentan a continuación para que otros educadores, que así lo deseen, puedan reproducir la intervención:

\section{Problema 2:}

En la construcción 1 , considere la función $f(x)=\operatorname{sen}(x)$ y los valores $a=-1$ y $b=2$. Use la opción de arrastre para mover el punto manipulando con sus dedos la pantalla del dispositivo móvil.

1.

2 .

2.1. ¿Es posible ubicar a $C$ entre $A$ y $B$ de tal manera que la inclinación de la recta $L_{1}$ sea igual a la inclinación del segmento $\overline{D F}$ ?

2.2. Calcule la derivada $f$ de en el valor $x=c$ para el que usted cree que recta $L_{1}$ y segmento $\overline{D F}$ tienen la misma inclinación.

2.3. Pregunte a sus compañeros si consideraron el mismo valor para $c$.

2.4. ¿Cuál es la pendiente de la recta que contiene al segmento $\overline{D F}$ ?

2.5. Comente con los compañeros sus hallazgos.

2.6. ¿Es posible ubicar a fuera del intervalo $[a, b]$ de tal manera que la inclinación de la recta sea igual a la inclinación del segmento $\overline{D F}$ ?

2.7. Comente con los compañeros sus hallazgos.

2.8. Enuncie una propiedad que defina la relación existente entre el valor $c$, el segmento $\overline{D F}$ y la recta $L_{1}$.

\section{Problema 3:}

Mediante la interacción con la construcción 1, desde sus dispositivos móviles, explore otras funciones diferentes a $f(x)=\operatorname{sen}(x)$ en intervalos diferentes de su dominio:

1.

2.

3.

3.1. Considere la función $f(x)=e^{\mathrm{x}} \mathrm{y}$ el intervalo $[a, b]=[-1,1]$. ¿Existen valores $c$ fuera del intervalo $(-1,1)$ que satisfagan la propiedad planteada en el punto $2.8 ?$ ¿Y existe al menos un $c$ en el intervalo que la cumpla?

3.2. Considere la función $f(x)=x^{2}+1$ y el intervalo $[a, b]=[-1,2]$. ¿Existen valores $c$ fuera del intervalo $(-1,2)$ que satisfagan la propiedad planteada en el punto 2.8 ? ¿Y existe al menos un $c$ dentro del intervalo que la cumpla? 
3.3. Comente con los compañeros sus hallazgos.

3.4. ¿Considera usted que para toda función $f(x)$ existe un $c \in(a, b)$ para el que la inclinación del segmento $\overline{F E}$ es igual a la inclinación de la recta $L_{1}$ ? Para responder a esta pregunta proponga algunas funciones que considere interesantes para poder generalizar lo observado.

3.5. Para los puntos 2.1 y 2.2 evalúe la derivada de $f$ en $x=c$, luego calcule la pendiente de la recta que contiene a $\overline{F E}$. ¿Qué concluye de estos resultados?

3.6. Teniendo en cuenta sus observaciones hechas en los problemas 1 y 2 , enuncie nuevamente la propiedad del punto 2.8 que involucra el valor $c$ el intervalo $[a, b]$ y la igualdad en inclinación de $\overline{F E}$ y $L_{1}$.

\section{Problema 4:}

Mediante la interacción con la construcción 1, desde sus dispositivos móviles, explore la función $f(x)=[x]$ (parte entera de $x$ ).

1.

2.

3.

4 .

4.1. En el punto 3.6 se enunció una propiedad que relaciona la pendiente de la recta $L_{1}$, la inclinación del segmento $\overline{F E}$ y el valor $c$. Se cumple esa propiedad considerando la función $f(x)=[x]$ en el intervalo $[a, b]=[1.3,1.8]$. Justifique su respuesta.

4.2. Nota: En la aplicación móvil de GeoGebra la sintaxis para $f(x)=[x]$ es $f(x)=f l o o r(x)$.

4.3. Comente sus hallazgos con los compañeros.

4.4. Responda el mismo enunciado de la pregunta 4.1, esta vez, considerando el intervalo $[a, b]=[1.3,2.8]$.

4.5. ¿Cree usted que la continuidad de la función $f(x)$ en el intervalo $[a, b]$ "cerrado" afecta en la conclusión de la propiedad enunciada en el punto 3.6?, argumente su respuesta.

4.6. ¿Cree usted que la continuidad de la función $f(x)$ en el intervalo $(a, b)$ "abierto" afecta en la conclusión de la propiedad enunciada en el punto 3.6?, argumente su respuesta. Para esto considere distintos valores para los extremos del intervalo.

4.7. Comparta sus ideas con los compañeros de grupo.

4.8. Considera necesario modificar la propiedad enunciada en el punto 3.6 teniendo en cuenta la continuidad de la función. Argumente su respuesta.

4.9. De ser afirmativa su respuesta al punto 4.7 modifique la propiedad enunciada en el punto 3.6. 


\section{Problema 5:}

Mediante la interacción con la construcción 1, desde sus dispositivos móviles, explore la función $f(x)=\sqrt{ }(|x|$ :

1.

2 .

3.

4

5.

5.1. En el punto 4.8 se enunció una propiedad que relaciona la pendiente de la recta $L_{1}$, la inclinación del segmento $\overline{F E}$ y el valor $c$. Se cumple esa propiedad considerando la función en el intervalo $[a, b]=[-1,1]$. Justifique su respuesta.

5.2. Comente sus hallazgos con los compañeros.

5.3. Responda el mismo enunciado de la pregunta 5.1, esta vez, considerando el intervalo $[a, b]=[-0.5,1]$.

5.4. Responda el mismo enunciado de la pregunta 5.1, esta vez, considerando el intervalo $[a, b]=[-0.5,1]$ y la función $f(x)=|x|$.

5.5. ¿Cree usted que la diferenciabilidad de la función $f(x)$ se debe dar en el intervalo $(a, b)$ "abierto" para que la conclusión de la propiedad enunciada en el punto 4.8 se cumpla?, argumente su respuesta.

5.6. ¿Cree usted que la diferenciabilidad de la función $f(x)$ se debe dar en el intervalo $[a, b]$ "cerrado" para que la conclusión de la propiedad enunciada en el punto 4.8 se cumpla?, argumente su respuesta a partir de considerar $f(x)=\sqrt{ }|\mathrm{x}|$ en el intervalo $[0,1]$.

5.7. Comparta sus ideas con los compañeros de grupo.

5.8. ¿Considera necesario modificar la propiedad enunciada en el punto 4.8 teniendo en cuenta la diferenciabilidad de la función $f$ en el intervalo $[a, b]$ ? Argumente su respuesta.

5.9. De ser afirmativa su respuesta al punto 5.8 modifique la propiedad enunciada en el punto 4.8.

\section{Problema 6:}

Complemente la construcción, haciendo uso de las herramientas que ofrece la App de GeoGebra para lograr que sea visible la inclinación del segmento $\overline{F E}$ y la pendiente de la recta $L_{1}$, una vez que se logre hacer ese cambio, responda las siguientes preguntas: 
1.

2.

3.

4.

5.

6.

6.1. Verifique que su construcción es válida considerando varios casos particulares para $f, a$ y $b$. Describa algunas propiedades observadas.

6.2. Si la inclinación del segmento $\overline{F E}$ es la razón de cambio promedio de la función $f(x)$ en el intervalo $[a, b]$ y la pendiente de $L_{1}$ es la razón de cambio puntual de $f$ en $x=c$, enuncie una propiedad general que relacione la razón de cambio promedio de $f(x)$ en el intervalo $[a, b]$ y su razón de cambio puntual o derivada en el intervalo $(a, b)$.

6.3. Comente su planteamiento con los compañeros.

6.4. ¿Es posible que esta propiedad se cumpla para toda función $f(x)$ que se elija? Justifique su respuesta.

6.5. De ser negativa su respuesta al numeral 6.4 dé algunos ejemplos de funciones que no la cumplen.

6.6. Justifiquen esta propiedad con base en las propiedades y definiciones construidas.

\section{Problema 7:}

La posición de una partícula que se mueve en línea recta con una aceleración constante (MRUA) está dada por la ecuación (2):

$$
x(t)=x_{0}+v_{0} t+\frac{1}{2} a t^{2}
$$

Donde $x_{0}$ es la posición inicial, $v_{0}$ es la velocidad inicial y $a$ la aceleración.

1.

2 .

3.

4.

5.

6.

7. 
7.1. La velocidad promedio de la partícula está dada por la ecuación (3), si se sabe que $x_{0}=0.4 \mathrm{~m} ; v_{0}=0.2 \mathrm{~m} / \mathrm{s} ; a=0.5 \mathrm{~m} / \mathrm{s}^{2}$ ¿Cuál es la velocidad promedio de la partícula en el periodo entre $t_{\mathrm{i}}=1.2 \mathrm{~s}$ y $t_{\mathrm{f}}=3 \mathrm{~s}$ ?

$$
v_{\text {prom }}=\frac{x\left(t_{f}\right)-x\left(t_{i}\right)}{t_{f}-t_{i}}=\frac{\Delta x}{\Delta t}
$$

7.2. Si la velocidad de la partícula en un instante $t$ está dada por la derivada de la posición evaluada en ese instante, es decir $v(t)=x^{\prime}(t)$. ¿Existe algún valor $t_{\mathrm{p}} \in(1.2,3)$ para el que $v_{\text {prom }}[1.2,3]=\mathrm{v}\left(t_{\mathrm{p}}\right)$ ? ¿Cuál? (Tome los valores del punto 7.1)

7.3. ¿Se puede ver esta situación, como un ejemplo aplicado del teorema del valor medio para derivadas? Justifique su respuesta.

7.4. ¿Cumple la función de posición con las hipótesis del teorema del valor medio?

7.5. ¿Se pueden generalizar sus hallazgos a cualquier función de posición de un MRUA? Para un proceso de observación modifique los valores $x_{0}, v_{0}$ y $a$ de en la aplicación móvil de GeoGebra, así como el intervalo de tiempo.

7.6. Escriba una conjetura que generalice lo observado en el punto 5.5 de acuerdo con el contexto del MRUA.

7.7. Responda la pregunta 1.2 del problema 1, esta vez aplicando la propiedad enunciada en el numeral 6.2. Justifique su respuesta con base en las definiciones y propiedades construidas.

En el diseño de esta secuencia didáctica, se propuso como hipótesis que los estudiantes debían detectar por sí mismos el invariante, es decir, que mediante la manipulación de los objetos matemáticos en la ACGG a través del arrastre debían ver que cuando la función $f(x)$ es continua y diferenciable en $[a, b]$, existe $c \in(a, b)$ tal que (ecuación 4).

$$
\frac{f(b)-f(a)}{b-a}=f^{\prime}(c)
$$

\section{Resultados}

En esta sección, por facilidad, se denotan los estudiantes como $E_{1}, E_{2}, E_{3}, E_{4}, E_{5}$, para describir los procesos de conjeturación del TVMD observados en cada uno de ellos:

Para la solución del Problema 1, nadie referenció el TVMD, sin embargo, sí se observa una predisposición de ellos por querer vincular la ACGG al desarrollo del proyecto de investigación. Como lo afirma $E_{4}$ :

Entre todo el grupo se concluyó que desconocemos las capacidades y todo lo que ofrece esta aplicación y que además en el aula de clases ayudaría en el aprendizaje y permitiría que entendiéramos mejor los temas o conceptos matemáticos y su aplicación. 
Sin embargo, para el problema 2 el estudiante $E_{2}$ logró relacionar una representación geométrica con la solución (Tabla 3a) de acuerdo con su interacción con la ACGG desde su dispositivo móvil, y agregó "Sí es posible que el punto $C$ arrastrándolo tenga la misma inclinación que la recta $L_{1}$, estando $C$ entre los puntos $A$ y $B$ ”. Por su parte, $E_{3}$ hizo una representación similar para la solución del problema (Tabla 3b) de acuerdo con las observaciones que tuvo en la ACGG, él afirmó: "es posible ubicar a fuera de ya que con la herramienta de deslizador ubicamos a fuera de ese intervalo y podemos observar que la recta $L_{1}$ es casi igual a la inclinación $\overline{D F}$.

TABLA 3.

Solución del problema 2 dada por $E_{1}$ y $E_{2}$

a. Representación hecha por $E_{2}$.

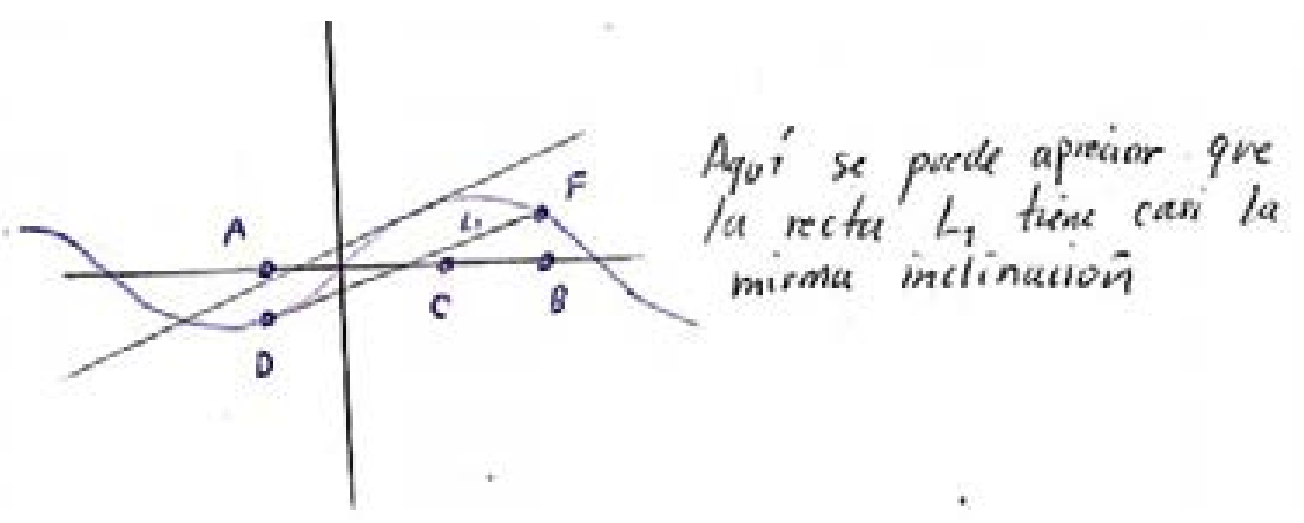

b. Representación hecha por $E_{3}$.

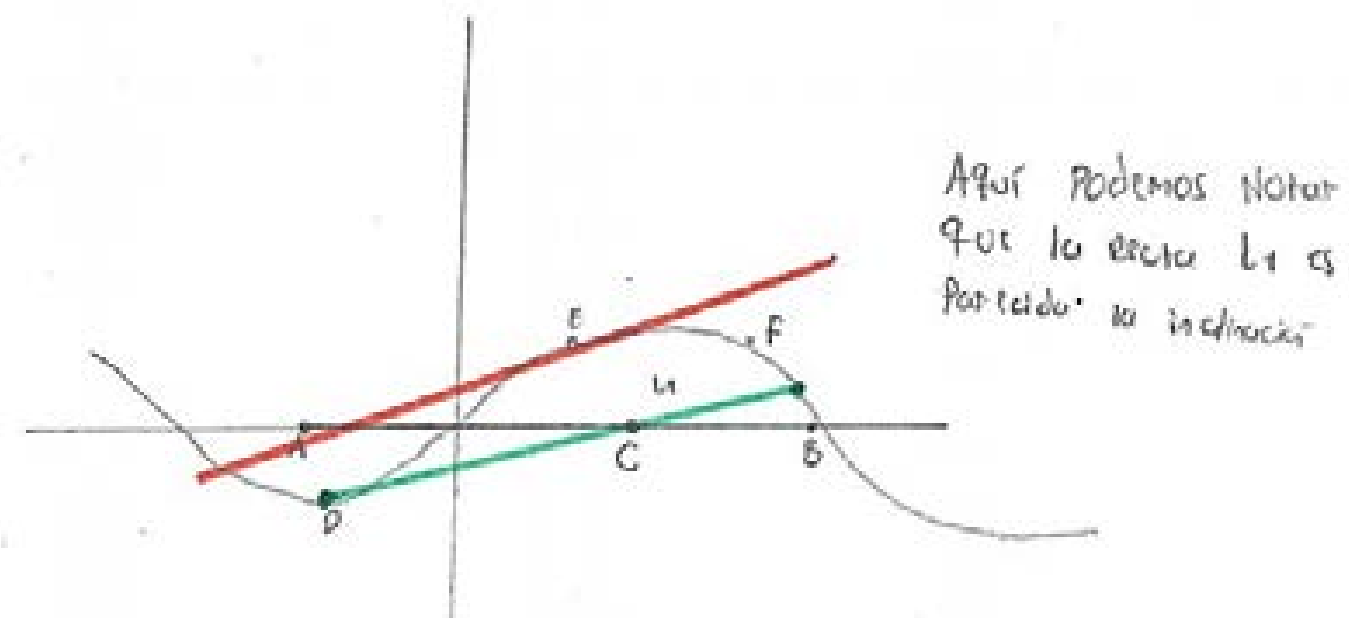

Fuente: Elaboración propia.

De manera similar $E_{1}$ y $E_{2}$ describen la misma observación identificando que existen valores en el dominio de la función $f(x)=\operatorname{sen}(x)$ tal que la pendiente de la recta $L_{1}$ es igual la inclinación del segmento $\overline{D F}$. No obstante, en aquel momento de la actividad ninguno, de los estudiantes pensó en la influencia de la continuidad o la diferenciabilidad de la función para hacer sus afirmaciones y tampoco generaron una proposición sino simplemente describían lo observado en la ACGG. 
En la Tabla 4 se muestra la evolución del estudiante $E_{1}$ por cada sesión del estudio de caso dentro del proceso de conjeturación del TVMD y un análisis de los resultados:

TABLA 4.

Evolución de E1 en el proceso de conjeturación del TVMD durante el estudio de caso

\begin{tabular}{|c|c|c|}
\hline Sesión & & Afirmación \\
\hline 1 & $\begin{array}{l}\text { "Yo puc } \\
\text { mueva } \\
\text { sen(x) } \\
\text { mostra }\end{array}$ & $\begin{array}{l}\text { e observar que el punto c siempre va influenciar a la recta tangente ya que cada vez que se } \\
C \text { la recta tangente también se va a mover y va a mostrar todas las tangentes de la función } \\
\text { ue serían las razones promedio puntual mientras que el segmento } \overline{D F} \text { siempre nos va a } \\
\text { c la razón de cambio promedio". }\end{array}$ \\
\hline 2 & $\begin{array}{l}\text { "Para c } \\
\text { segmen } \\
\text { c afuer }\end{array}$ & $\begin{array}{l}\text { ualquier función el punto c dentro del intervalo }(A, B) \text { hace que la recta tangente y el } \\
\text { to tenga la misma inclinación, pero para las funciones } \cos (x) \text {, sen }(x) \text { y lineales existe un punto } \\
\text { a del intervalo }(A, B) \text { que satisfacen la propiedad, mientras que en otras funciones no". }\end{array}$ \\
\hline 3 & $\begin{array}{l}\text { "Para t } \\
\text { propied }\end{array}$ & $\begin{array}{l}\text { oda función continua se cumple la propiedad, pero para una función discontinua esta } \\
\text { ad no se cumple". }\end{array}$ \\
\hline 4 & $\begin{array}{l}\text { "Para o } \\
\text { para qu } \\
\text { función }\end{array}$ & $\begin{array}{l}\text { ue se satisfaga esta propiedad la función tiene que ser continua y que tenga puntos derivables } \\
\text { e la propiedad se satisfaga si no hay puntos derivables la propiedad no se cumplirá para la } \\
\text { continua". }\end{array}$ \\
\hline \multirow{3}{*}{5} & Sesscen & Anrmacace \\
\hline & 1 & 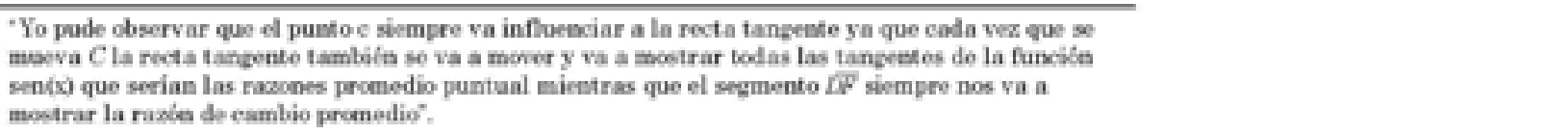 \\
\hline & 2 & 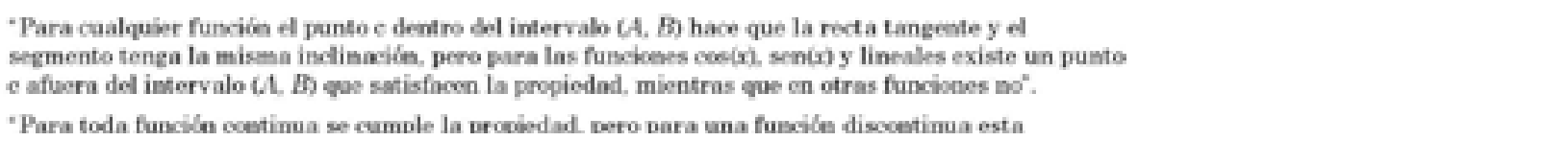 \\
\hline
\end{tabular}

Fuente: Elaboración propia.

En el caso de $E_{1}$, como se observa en la Tabla 4, se da un cambio del estado de descripción al de enunciación de la conjetura que, a pesar de no ser muy precisa y tener errores de enunciado, se evidencia reconocimiento de la invariante mediante arrastre, observó que bajo condiciones de continuidad y diferenciabilidad de $f(x)$ en $[a, b]$ se cumple el TVMD. Por su parte $E_{2}$, se observa en la Tabla 5.

TABLA 5.

Evolución de E2 en el proceso de conjeturación del TVMD.

\begin{tabular}{|c|c|c|}
\hline Sesión & & Afirmación \\
\hline 1 & $\begin{array}{l}\text { "Una } \\
\text { tenga } \\
\text { segme }\end{array}$ & $\begin{array}{l}\text { osible relación que puede existir es que encontrar un valor exacto de } C \text { de tal manera que } \\
\text { la misma inclinación que el segmento } D F \text { y que } L_{1} \text { depende de la posición de C. También que el } \\
\text { ato } D F \text { representa la razón de cambio promedio y el punto c la razón de cambio puntual". }\end{array}$ \\
\hline 2 & "Para & $\begin{array}{l}\text { toda función } f(x) \text { siempre habrá un punto c que sea paralela o tenga la misma inclinación a la } \\
\text { ". }\end{array}$ \\
\hline 3 & $\begin{array}{l}\text { "Para } \\
\text { interv } \\
\text { dentro }\end{array}$ & $\begin{array}{l}\text { toda función } f(x) \text { siempre habrá un punto } C \text { por fuera, o tanto por fuera como por dentro del } \\
\text { alo }[a, b] \text { que cumpla una continuidad, siempre tendrá la misma inclinación, mientras que } \\
\text { del intervalo }[a, b] \text { es discontinua no se cumplirá la propiedad ". }\end{array}$ \\
\hline 4 & "Para & toda función $f(x)$ que sea continua y derivable en cualquier punto, se cumple la propiedad". \\
\hline \multirow{4}{*}{5} & Sesicie & Afirmasín \\
\hline & 1 & 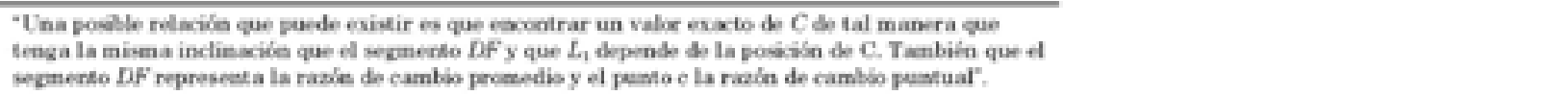 \\
\hline & 2 & 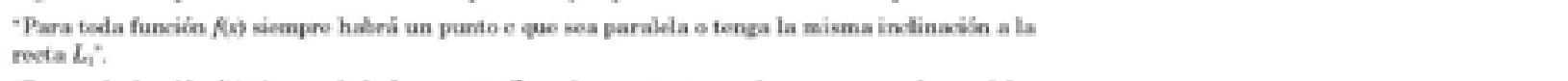 \\
\hline & & 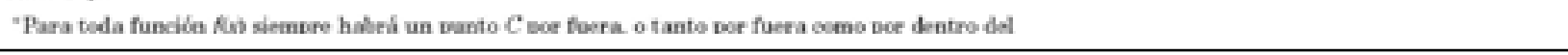 \\
\hline
\end{tabular}

Fuente: Elaboración propia. 
De acuerdo con la Tabla 5, en el caso del estudiante, se evidencia el reconocimiento del invariante que bajo condiciones de continuidad y diferenciabilidad de $f(x)$ en $[a, b]$ se mantiene la conclusión del TVMD (Tabla 3). Es notable como logró pasar de describir lo que observó en la ACGG a plantear una conjetura del TVMD.

TABla 6.

Evolución de E3 en el proceso de conjeturación del TVMD

\begin{tabular}{|c|c|c|}
\hline Sesión & & Afirmación \\
\hline 1 & $\begin{array}{l}\text { "Depe } \\
\text { segme }\end{array}$ & $\begin{array}{l}\text { ndiendo de c la recta tangente varía, pero llega un momento en el que existe una relación del } \\
\text { nto } \overline{D F} \text { con respecto a la recta } L_{1} \text { y muestra los valores medios". }\end{array}$ \\
\hline 2 & $\begin{array}{l}\text { "Esta } \\
\text { interv } \\
\text { puede }\end{array}$ & $\begin{array}{l}\text { propiedad antes mencionada sirve para poder analizar y concluir que existe un c dentro del } \\
\text { alo }[a, b] \text { que pueda cumplir esta propiedad, pero por fuera de este intervalo la propiedad no se } \\
\text { cumplir". }\end{array}$ \\
\hline 3 & "Dent & $\begin{array}{l}\text { co de tal intervalo la función es continua y cumple la propiedad, pero tiene que ser en un } \\
\text { alo específico que satisfaga esta propiedad”. }\end{array}$ \\
\hline 4 & $\begin{array}{l}\text { "En el } \\
\text { la pro }\end{array}$ & $\begin{array}{l}\text { punto } 4.8 \text { concluimos que cumplía la propiedad solo si era continua, pero nos dimos cuenta que } \\
\text { jiedad tiene que ser continua y ser derivable en todos los puntos para que cumpla”. }\end{array}$ \\
\hline \multirow[b]{2}{*}{5} & $\frac{\infty \sin }{1}$ & 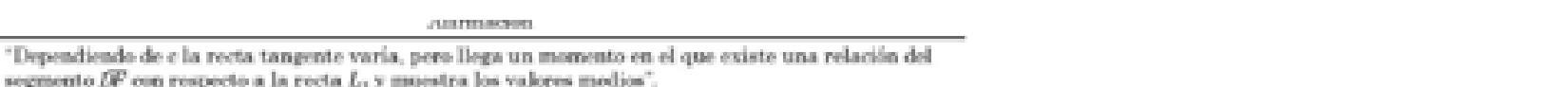 \\
\hline & 2 & 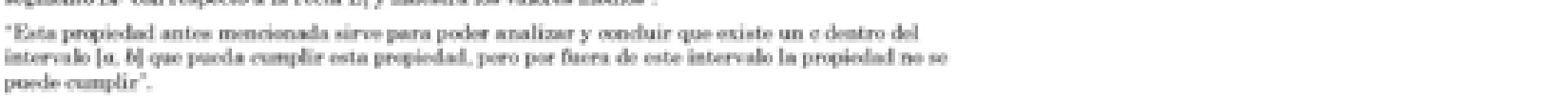 \\
\hline
\end{tabular}

Fuente: Elaboración propia.

El estudiante, menciona las condiciones de continuidad del TVMD, y establece la relación que existe entre la razón de cambio promedio y la razón de cambio puntual de $f(x)$. Aunque no expresa en un lenguaje matemático sofisticado, en sus palabras se aprecia una secuencia lógica de implicación (Tabla 6).

TABLA 7.

Evolución de E4 en el proceso de conjeturación del TVMD

\begin{tabular}{|c|c|c|}
\hline Sesión & & Afirmación \\
\hline 1 & $\begin{array}{l}\text { "El valo } \\
\text { segmen } \\
\text { seno; en } \\
\text { misma }\end{array}$ & $\begin{array}{l}r \text { de } C \text { al ser una recta tangente que se mueve por todos los puntos de la función seno y el } \\
\text { o está anclado a dos puntos de una cresta y esta cresta al ser igual a todas las de la función } \\
\text { tonces esa igualdad permitiría que } L_{1} \text { en distintos puntos de diferentes crestas adopte la } \\
\text { nclinación que el segmento". }\end{array}$ \\
\hline 2 & $\begin{array}{l}\text { "Para to } \\
\text { fuera de }\end{array}$ & $\begin{array}{l}\text { da función siempre habrá un c que haga que } \overline{F E}=L_{1} \text {; entonces para encontrar puntos } C \\
\text { lintervalo dependerá del tipo de función ". }\end{array}$ \\
\hline 3 & $\begin{array}{l}\text { "Para tc } \\
\text { habrá u } \\
\text { disconti } \\
\text { función }\end{array}$ & $\begin{array}{l}\text { da función dentro de un intervalo }[a, b] \text { que esté en continuidad con la función al menos } \\
\text { n punto } C \text { que haga que el segmento sea igual a la inclinación de } L_{1} \text {; pero si la función es } \\
\text { nua la propiedad no se cumple; el intervalo tendría que estar en una parte continua de la } \\
\text { para que esta se cumpla". }\end{array}$ \\
\hline 4 & $\begin{array}{l}\text { "Para to } \\
\text { del inte. } \\
\text { interval }\end{array}$ & $\begin{array}{l}\text { da función continua que sea derivable en todo su dominio, siempre habrá un punto C dentro } \\
\text { cvalo }[a, b] \text { hará que } L_{1} \text { tenga la misma inclinación del segmento } \overline{F E} \text { no importa el tipo de } \\
\text { o cerrado o abierto". }\end{array}$ \\
\hline \multirow{3}{*}{5} & somen & 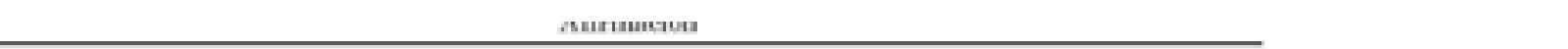 \\
\hline & 1 & 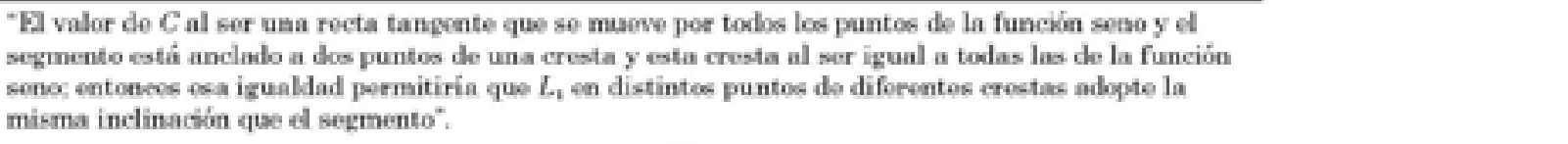 \\
\hline & 2 & $\begin{array}{l}\text { "Para todn funcisin siempre habrí un e que hagn que } F \overline{F E}=L_{4} \text {; enteners parn encontrar puntes } C \\
\text { fuera del intervalo dependerá del tipo de función? }\end{array}$ \\
\hline
\end{tabular}

Fuente: Elaboración propia. 
De acuerdo con la información recopilada en la Tabla 7, el estudiante $E_{4}$ usó el conector lógico "para todo" en su proposición y se ve que detectó el invariante por medio de la interacción con la ACGG. Él observó que si la función $f(x)$ es continua y diferenciable, sin importar la función, se podía encontrar el valor $c$ en $[a, b]$ para el que la razón de cambio promedio es igual a la razón de cambio puntual.

TABLA 8.

Evolución de E5 en el proceso de conjeturación del TVMD

\begin{tabular}{|c|c|c|}
\hline Sesión & & Afirmación \\
\hline 1 & \multicolumn{2}{|r|}{$\begin{array}{l}\text { "Realmente no noté importancia lo que varía c, hace variar y notar las tangentes, a la hora de } \\
\text { interactuar no me quedó muy claro que podría responder en la pregunta". }\end{array}$} \\
\hline 2 & \multicolumn{2}{|r|}{$\begin{array}{l}\text { "La parte de las funciones discontinuas que afectará enormemente si el intervalo se sale de control } \\
\text { no cumplirá lo establecido que se desarrolló en el punto } 3.6 \text { ". }\end{array}$} \\
\hline 3 & \multicolumn{2}{|c|}{ El estudiante no asistió a esta sesión de clase. } \\
\hline 4 & \multicolumn{2}{|r|}{$\begin{array}{l}\text { "La función debe ser continua, derivable sin importar sus tipos de intervalo, para tener en cuenta la } \\
\text { propiedad". }\end{array}$} \\
\hline \multirow{5}{*}{5} & Sesibn & Afirmarión \\
\hline & 1 & 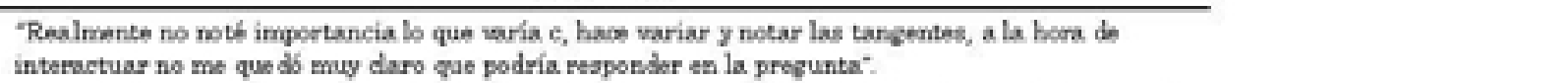 \\
\hline & 2 & 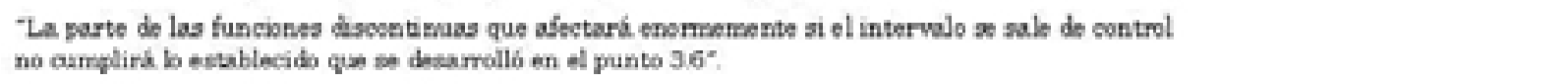 \\
\hline & 3 & El estudiante no asistí a esta sesiba de clase. \\
\hline & 4 & 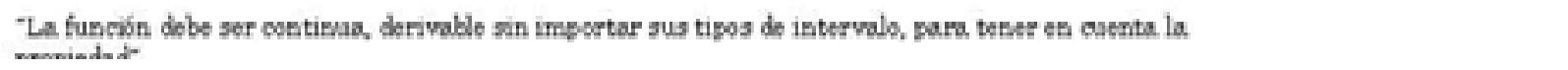 \\
\hline
\end{tabular}

Fuente: Elaboración propia.

Como se observa en la Tabla 8, el estudiante no muestra una identificación clara de los invariantes involucrados en el TVMD, tampoco enuncia una conjetura de manera coherente ni llega a la descripción de observaciones en la ACGG.

\section{DiscUsióN}

Coherentemente con Mariotti (2006) refiriéndose a la función de arrastre de los programas de geometría dinámica, en este estudio se observa que el arrastre proporciona a los estudiantes una fuerte evidencia perceptiva, tanto en el orden visual como kinestésico del TVMD, no obstante, los llevó a considerar la exploración en la ACGG como suficiente para garantizar la veracidad del teorema, esto hace que para los estudiantes sea innecesario o inimaginable hacer una demostración del fenómeno matemático desde una construcción axiomática y esto se puede derivar en un problema.

En la misma línea de Rocha (2019), este estudio de caso, afirma que la prueba, o demostración matemática, puede tener varias funciones, incluidas la verificación, la explicación, la sistematización, el descubrimiento y la comunicación, todas estas importantes para los matemáticos. No obstante, en la población tomada para esta investigación, sólo se logró la función de verificación y las circunstancias en las que ocurrió este fenómeno se limitaron a resultados intuitivos desde la visualización en la ACGG mediante el arrastre, 
obteniendo como consecuencia que los estudiantes no percibieran el punto de la demostración. Sin embargo, y como señala Rocha (2019), la función de explicación es la más relevante porque permite una comprensión más profunda de los conceptos, función que parece estar relacionada con la noción de prueba simple; y esta investigación da cuentas de un avance en la explicación desde la visualización geométrica del TVMD a través de la interacción con la ACGG en dispositivos móviles.

De acuerdo con Ozdamli (2012), se puede decir que hay muchas posibilidades en el aprendizaje móvil para los estudiantes, sin embargo, debe discutirse el marco pedagógico del aprendizaje móvil, la integración de herramientas, los enfoques pedagógicos, las técnicas de evaluación y la formación del profesorado. Aunque el enfoque de aprendizaje móvil en este estudio de caso fue exitoso en cuanto a la explicación del TVMD, como trabajo futuro, se recomienda la investigación del aprendizaje móvil a mayor escala experimentalmente para una integración asertiva en los procesos de conjeturación. En el mismo sentido, este estudio proporciona evidencia que respalda lo dicho (Donaldson, 2011; Akour, 2011; AbuAl-Aish, Love, Hunaiti \& Al-masaeed, 2014; Huang, 2014), exhortando a las instituciones de educación superior a comprender, de forma adecuada, el uso del m-learning por parte de los estudiantes, además de los factores presentes en la utilización y la aceptación, por parte de los mismos, para planificar el desarrollo y la implementación de los sistemas de m-learning en los currículos universitarios.

\section{ConClusiones}

La investigación da cuenta del carácter mediador de las herramientas propias del m-learning en el contexto del aula de clase. El grupo de estudiantes logró detectar un invariante que les permitió conjeturar un teorema central del cálculo diferencial como es el TVMD, a partir de la interacción con las herramientas de geometría dinámica que ofrece la ACGG gracias al uso de las opciones de arrastre. Cuatro de los cinco estudiantes (a excepción de E5) lograron conjeturar que si una función $f(x)$ es continua y diferenciable en el intervalo $(a, b)$, entonces siempre habrá un $c \in(a, b)$ tal que $(f(b)-f(a)) /(b-a)=f^{\prime}(c)$. Evidenciar este hecho se configura como un hallazgo, es decir, este diseño de clase efectivamente facilitó el proceso de conjeturación y, en este sentido, la actividad fue de gran valor. Se podría afirmar que E4 planteó una conjetura usando conectores lógicos y un lenguaje matemático fluido, sin embargo, no se encontró evidencia significativa para asegurar que se logró un cambio en el proceso de demostración desde una perspectiva teórica ya sea en pruebas de tipo probatorio o de justificación.

Se evidenció un reconocimiento de la invariante pretendido con la ayuda del m-learning, hecho que ayudó en el paso hacia la conjeturación del TVMD, esto gracias a la combinación de elementos de cálculo infinitesimal con geometría dinámica que permite la ACGG desde dispositivos móviles. El proceso de justificación se dio con argumentos geométricos basados en la percepción visual y kinestésica apoyada por los dispositivos móviles, donde los estudiantes referían elementos visuales, que lograron gracias a la interacción con la ACGG y las herramientas de arrastre para visualizar los invariantes a favor de un avance hacia la conjeturación. 
Se identificó, además, la necesidad de estudiar las interacciones entre las matemáticas y las tecnologías digitales como la ACGG y el m-learning, en relación con el proceso de conjeturación, teniendo en cuenta la interacción entre la sintaxis, la semántica y la pragmática, siendo pertinente abrir nuevas vías de investigación al ayudar a identificar cuestiones en la lógica, las matemáticas y el m-learning, que pueden pasarse por alto o permanecer implícitas en el aula o fuera de ella, en particular cuando se apoya el proceso de conjeturación con el uso de dispositivos móviles.

Por último, los resultados de este proyecto de investigación permiten establecer que si bien las tecnologías digitales como la ACGG y el m-learning pueden proporcionar una poderosa herramienta de enseñanza y aprendizaje, no pueden impulsar un cambio significativo por sí solos; en consecuencia, para que se adopte ampliamente esta iniciativa debe hacer parte de un esfuerzo amplio y sistemático para incidir sobre los procesos de conjeturación en matemáticas. Esta investigación, proporciona una mirada a los beneficios potenciales de la tecnología digital como la ACGG sobre los procesos de conjeturación en matemáticas y, aporta evidencia necesaria para comprender mejor cómo el $\mathrm{m}$-learning puede ayudar a la generación de procesos más efectivos en el desarrollo de conjeturas por parte de los sujetos que aprenden.

\section{Agradecimientos}

Los autores agradecen a la Fundación Universitaria Los Libertadores por la financiación del proyecto de investigación y a los estudiantes y profesores de la Facultad de Ingeniería y Ciencias Básicas que facilitaron el desarrollo del mismo.

\section{REFERENCIAS}

Abu-Al-Aish, A., Love, S., Hunaiti, Z. \& Al-masaeed, S. (2014). Toward mobile learning deployment in higher education. International Journal of Mobile Learning and Organisation (IJMLO), 7(3/4), 253-276. https://doi.org/10.1504/IJMLO.2013.057165.

Akour, H. (2011). Determinants of mobile learning acceptance: An empirical investigation in higher education. [Ph.D. Dissertation]. Oklahoma State University, Small city, USA. Available: https://www.proquest.com/docview/610058264

Al-Emran, M., Mezhuyev, V. \& Kamaludin, A. (2018). Technology Acceptance Model in M-learning context: A systematic review. Computers \& Education, 125, 389-412. https://doi.org/10.1016/j.compedu.2018.06.008

Baccaglini-Frank, A. (2019). Dragging, instrumented abduction and evidence, in processes of conjecture generation in a dynamic geometry environment. ZDM Mathematics Education, 51, 779-791. https://doi.org/10.1007/s11858-019-01046-8

Boero, P., Fenaroli, G. \& Guala, E. (2018). Mathematical Argumentation in Elementary Teacher Education: The Key Role of the Cultural Analysis of the Content. In: Stylianides A., Harel G. (eds), Advances in Mathematics Education Research on Proof and Proving, (pp. 49-67). Cham: Springer. https://doi.org/10.1007/978-3-31970996-3_4 
Cabri Geometry (versión 2.1.1) [software de geometría]. Grenoble: Cabrilog. Disponible en https://cabri.com/es/

Camargo, L., Samper, C. \& Perry, P. (2007). Cabri's role in the task of proving within the activity of building part of an axiomatic system. In: D. Pitta-Pantazi \& G. Philippou (Eds.), Proceedings of the Fifth Congress of the European Society for Research in Mathematics Education (pp. 571-580). Larnaca: CERME. Recuperado de http:// funes.uniandes.edu.co/927/1/2007Pr-CamargoCabri.pdf

Cheema, S., Gulwani, S. \& LaViola, J. (may, 2012). QuickDraw: improving drawing experience for geometric diagrams. In: J. Konstan, Proceedings of the SIGCHI Conference on Human Factors in Computing Systems (pp. 1037-1064). Association for Computing Machinery, New York, United States. https://doi.org/10.1145/2207676.2208550

Creswell, J. W. (2017). Research design: Qualitative, quantitative, and mixed methods approaches. Thousand: Sage publications. Recuperado de http://www.drbrambedkarcollege.ac.in/sites/default/files/research-design-ceil.pdf

Crompton, H., Burke, D., Gregory, K. H. \& Grabe, C. (2016). The use of mobile learning in science: A systematic review. Journal of Science Education and Technology, 25, 149-160. https://doi.org/10.1007/s10956-015-9597-x

Donaldson, R. L. (2011). Student acceptance of mobile learning. [Ph.D. Dissertation]. The Florida State University, Tallahassee, USA. Recuperado de https://fsu.digital. flvc.org/islandora/object/fsu\%3A168891/datastream/PDF/view

Ehmann, M., Gerhauser, M., Miller, C. \& Wassermann, A. (2013). Sketchometry and jsxgraph-dynamic geometry for mobile devices. South Bohemia Mathematical Letters, 21(1), 1-7. Recuperado de http://home.pf.jcu.cz/ upvvm/2013/sbornik/clanky/09_ UPVM2013_Ehmann_et_al.pdf

Escuder, A. \& Furner, J. M. (2011). The Impact of GeoGebra in Math Teacher's Professional Development. In: International Conference on Technologies in Collegiate Mathematics (pp. 76-84). Denver: Pearson. Recuperado de http://archives.math. utk.edu/ICTCM/VOL23/S113/paper.pdf

Geogebra Calculadora Gráfica. (versión versión 6.0.619.0) [software de geometría]. Linz: ACGG. Disponible en https:/www.geogebra.org/graphing?lang=es

Hamidi, H. \& Chavoshi, A. (2018). Analysis of the essential factors for the adoption of mobile learning in higher education: A case study of students of the University of Technology. Telematics and Informatics, 35(4), 1053-1070. https://oi.org/10.1016/j. tele.2017.09.016

Hanna, G. (2018). Reflections on Proof as Explanation. In: Stylianides A., Harel G. (eds), Advances in Mathematics Education Research on Proof and Proving (pp. 3-18). Cham: Springer. https://doi.org/10.1007/978-3-319-70996-3_1

Hanna, G. (1995). Challenges to the importance of proof'. For the Learning of Mathematics 15(3), 42-49. Recuperado de https://flm-journal.org/Articles/7679867298F4 CBEABE82D0ABEB5EC.pdf 
Holmes, B. \& Gardner, J. (2006). E-Learning (Concepts and practice). London: SAGE Publications. http://dx.doi.org/10.4135/9781446212585

Huang, Y. (2014). Empirical Analysis on Factors Impacting Mobile Learning Acceptance in Higher Engineering Education. [Doctoral dissertation]. University of Tennessee, Knoxville, USA. Recuperado de https://trace.tennessee.edu/cgi/viewcontent. cgi?article $=3166 \&$ context $=$ utk_graddiss

Joo-Nagata, J., Martinez, F., García-Bermejo, J., \& García-Peñalvo, F. J. (2017). Augmented reality and pedestrian navigation through its implementation in m-learning and e-learning: Evaluation of an educational program in Chile. Computers \& Education, 111, 1-17. https://doi.org/10.1016/j.compedu.2017.04.003

Karimi, S. (2016). Do learners' characteristics matter? An exploration of mobile-learning adoption in self-directed learning. Computers in Human Behavior, 63, 769776. https://doi.org/10.1016/j.chb.2016.06.014

Karunakaran, S. S. (2018). The Need for "Linearity" of Deductive Logic: An Examination of Expert and Novice Proving Processes. In: Stylianides A., Harel G. (eds), Advances in Mathematics Education Research on Proof and Proving (pp. 171-183). Cham: Springer. https://doi.org/10.1007/978-3-319-70996-3_12

Leung, A., Baccaglini-Frank, A. \& Mariotti, M. A. (2013). Discernment in dynamic geometry environments. Educational Studies in Mathematics, 84(3), 439-460. https:// doi.org/10.1007/s10649-013-9492-4

Majerek, D. (2014). Applications of GeoGebra for Teaching Mathematics. Advances in Science and Technology Research Journal, 8(24), 51-54. https://doi. org/10.12913/22998624/567

Mariotti, M. A. (2015). Transforming Images in a DGS: The semiotic potential of the dragging tool for introducing the notion of conditional statement. In: S. Rezat, M. Sebastian, A. Hattermann \& Peter-Koop (Eds.), Transformation-A fundamental idea of mathematics education (pp. 155-172). New York: Springer. https://doi. org/10.1007/978-1-4614-3489-4_8

Mariotti, M. (2006). Prof and proving in mathematics education. In: A. Gutiérrez \& P. Boero (Eds.), Handbook of research on the psychology of mathematics education: Past, present and future (pp. 173-204). Rotterdam: Sense Publishers.

Mariotti, M. A. \& Pedemonte, B. (2019). Intuition and proof in the solution of conjecturing problems'. ZDM, 51, 759-777. https://doi.org/10.1007/s11858-019-01059-3

Ozdamli, F. (2012). Pedagogical framework of m-learning. Procedia - Social and Behavioral Sciences, 31, 927-931. https://doi.org/10.1016/j.sbspro.2011.12.171

Pedemonte, B. (2018). How Can a Teacher Support Students in Constructing a Proof? Advances in Mathematics Education Research on Proof and Proving (pp. 115-129). Cham: Springer. https://doi.org/10.1007/978-3-319-70996-3_8

Pedemonte, B. (2007). How can the relationship between argumentation and proof be analysed? Educational Studies in Mathematics, 66(1), 23-41. https://doi.org/10.1007/ s10649-006-9057-x 
Pegrum, M. (2014). Agendas for Mobile Learning. In, M. Pegrum, Mobile learning: Languages, literacies and cultures (pp 4-16). UK. Springer. Recuperado de https:// link.springer.com/chapter/10.1057/9781137309815_2

Rocha, H. (2019). Mathematical proof: from mathematics to school mathematics. Philosophical Transactions of the Royal Society A: Mathematical, Physical and Engineering Sciences, 377(2140), 1-12. https://doi.org/10.1098/rsta.2018.0045

Sánchez, J. C., Olmos, S. \& Garcí-Peñalvo, F. (november, 2013). Mobile Learning: Tendencies and lines of research. In, F. García-Peñalvo (ed.), Proceedings of the first international conference on technological ecosystem for enhancing multiculturality (pp. 473-480). Association for Computing Machinery-ACM, New York, USA. https://doi.org/10.1145/2536536.2536609

Sánchez-Prieto, J. C., Olmos-Migueláñez, S. \& García-Peñalvo, F. (2016). Informal tools in formal contexts: Development of a model to assess the acceptance of mobile technologies among teachers. Computers in Human Behavior, 55(Part A), 519-528. https://doi.org/10.1016/j.chb.2015.07.002

Selaković, M., Marinković, V. \& Janičić, P. (2019). New dynamics in dynamic geometry: Dragging constructed points. Journal of Symbolic Computation, 97, 3-15. https:// doi.org/10.1016/j.jsc.2018.12.002

Velichova, D. (2011). Interactive Maths with GeoGebra. International Journal of Emerging Technologies in Learning (IJET), 6(S1), 31-35. Available: https://online-journals.org/index.php/i-jet/article/view/1620

Wassie, Y. A. \& Zergaw, G. A. (2019). Some of the Potential Affordances, Challenges and Limitations of Using GeoGebra in Mathematics Education. Eurasia Journal of Mathematics, Science and Technology Education, 15(8), 1-11. https://doi. org/10.29333/ejmste/108436

Vladimir Ballesteros Ballesteros es profesor universitario colombiano, dedicado a la Educación Matemática y la Educación en Ciencias. Actualmente, se desempeña como Decano de la Facultad de Ingeniería y Ciencias Básicas de la Fundación Universitaria Los Libertadores (Colombia). https://orcid.org/0000-0002-6920-789X

Óscar Iván Rodríguez Cardoso es nacido en Colombia, formado en Colombia. Licenciado en matemáticas de la Universidad Pedagógica Nacional (Colombia). Especialista en Matemática Aplicada de la Universidad Sergio Arboleda (Colombia). Magíster en Educación de la Fundación Universitaria Los Libertadores (Colombia). Aspirante a doctor en Educación en Ciencias de la Universidad Distrital Francisco José de Caldas (Colombia). Profesor de matemáticas y estadística en la Fundación Universitaria los Libertadores. https://orcid.org/0000-0003-1203-4999 
Sébastien Lozano Forero es de origen canadienes, formado en Colombia. Matemático de la Universidad Nacional (Colombia). Mágister en Estadística de la Universidade Federal de Pernambuco (Brasil). Especialista en Estadística Aplicada de la Fundación Universitaria Los Libertadores (Colombia). Profesor de Estadística en la Universidad del Rosario (Colombia) y la Fundación Universitaria los Libertadores. Consultor estadístico del Instituto Nacional de Salud. https://orcid.org/0000-0002-9551-165X 